\title{
Decreased Total Antioxidant Activity in Major Depressive Disorder Patients Non-Responsive to Antidepressant Treatment
}

\author{
Song-Eun Baek', Gyoung-Ja Lee ${ }^{2}$, Chang-Kyu Rhee ${ }^{2}$, Dae-Young Rho', \\ Do-Hoon Kim', Sun Huh ${ }^{3}$, and Sang-Kyu Lee ${ }^{1}$ \\ 1Department of Psychiatry, Hallym University Chuncheon Sacred Hospital, College of Medicine, Hallym University, Chuncheon, Republic of Korea \\ ${ }^{2}$ Nuclear Materials Development Division, Korea Atomic Energy Research Institute, Daejeon, Republic of Korea \\ ${ }^{3}$ Department of Parasitology, College of Medicine, Hallym University, Chuncheon, Republic of Korea
}

Objective This study aimed to evaluate the total antioxidant activity (TAA) in patients with major depressive disorder (MDD) and the effect of antidepressants on TAA using a novel potentiometric method.

Methods Twenty-eight patients with MDD and thirty-one healthy controls were enrolled in this study. The control group comprised 31 healthy individuals matched for gender, drinking and smoking status. We assessed symptoms of depression using the Hamilton Depression Rating Scale (HAMD) and the Beck Depression Inventory (BDI). We measured TAA using potentiometry. All measurements were made at baseline and four and eight weeks later.

Results There was a significant negative correlation between BDI scores and TAA. TAA was significantly lower in the MDD group than in controls. When the MDD group was subdivided into those who showed clinical response to antidepressant therapy (response group) and those who did not (non-response group), only the non-response group showed lower TAA, while the response group showed no significant difference to controls at baseline. After eight weeks of antidepressant treatment, TAA in both the response and non-response groups was similar, and there was no significant difference among the three groups.

Conclusion These results suggest that the response to antidepressant treatment in MDD patients might be predicted by measuring TAA.

Psychiatry Investig 2016;13(2):222-226

Key Words Major depressive disorder, Total antioxidant activity, Antidepressant treatment.

\section{INTRODUCTION}

Depression is a highly debilitating disease that impacts negatively on quality of life, cognitive function, and morbidity and mortality. ${ }^{1-3}$ Its pathogenesis is multifactorial and includes genetic factors, the monoamine deficiency hypothesis, dysregulation of the hypothalamic-pituitary-adrenal axis, altered glutamatergic neurotransmission, reduced GABAergic neurotransmission and increased inflammatory cytokine circulation. ${ }^{4}$ Recently, it has been suggested that major depression is related to the induction of the oxidative stress pathway and

Received: May 21, 2015 Revised: July 9, 2015

Accepted: July 9, 2015 Available online: October 19, 2015

$\triangle$ Correspondence: Sang-Kyu Lee, MD, PhD

Department of Psychiatry, Hallym University Chuncheon Sacred Hospital, College of Medicine, Hallym University, 77 Sakju-ro, Chuncheon 24253, Republic of Korea

Tel: +82-33-240-5174, Fax: +82-33-244-0317, E-mail: skmind@hallym.ac.kr

(a) This is an Open Access article distributed under the terms of the Creative Commons Attribution Non-Commercial License (http://creativecommons.org/licenses/by$\mathrm{nc} / 3.0$ ) which permits unrestricted non-commercial use, distribution, and reproduction in any medium, provided the original work is properly cited. to a decreased antioxidant status. ${ }^{5}$ Patients diagnosed with major depressive disorder (MDD) have been shown to have increased levels of reactive oxygen species (ROS) and reactive nitrogen species (RNS), including peroxide and nitric oxide $(\mathrm{NO}),{ }^{5}$ and reduced brain glutathione peroxidase (GSH-Px) levels. ${ }^{6}$ These free radicals modify cellular macromolecules (i.e., proteins, DNA, and lipids) and affect cellular functions, ${ }^{7}$ including apoptosis or necrosis. ${ }^{8-10}$ Among the body organs, the brain is especially sensitive to oxidative damage, given its requirement for large amounts of oxygen and the production of free radicals. ${ }^{11,12}$ In this context, there is a current hypothesis that suppressing pro-inflammatory cytokines and ROS/RNS production, or enhancing antioxidant defense, are therapeutic mechanisms of antidepressants. ${ }^{13}$

Previous studies have evaluated various oxidative and antioxidative markers to investigate oxidative stress. For example, superoxide dismutase (SOD), GSH-Px and catalase (CAT) activities, malondialdehyde (MDA) and NO levels, bilirubin, albumin, uric acid, and vitamins $\mathrm{A}, \mathrm{C}$, and $\mathrm{E}$ have been measured. ${ }^{5,6,14-18}$ 
Serum concentrations of various oxidant species can be measured; however, the measurements are time-consuming, laborintensive, and costly, and require specific techniques. ${ }^{19}$ For these reasons, recent studies have used measures of total antioxidant response (TAR) such as total antioxidant capacity (TAC), total antioxidant activity (TAA), or oxidative stress index (OSI). ${ }^{18,20}$ These methods can provide information on an individual's overall oxidant status. ${ }^{21}$ Among them, potentiometry utilizing redox reactions in a $\left[\mathrm{Fe}(\mathrm{CN})_{6}\right] 3-/\left[\mathrm{Fe}(\mathrm{CN})_{6}\right] 4$ - redox-reagent solution has been considered as a simple, highly sensitive, fast, and inexpensive method for evaluating TAA in samples such as drinks, food, and blood. ${ }^{22}$ We have recently evaluated the feasibility of TAA evaluation using potentiometry in MDD patients. ${ }^{23}$

To our knowledge, several studies have investigated the impact of antidepressant treatment on the TAR in patients with MDD. ${ }^{14,15,18}$ Sarandol et al. ${ }^{18}$ found no significant change in TAC after six weeks of antidepressant treatment. Cumurcu et al. ${ }^{15}$ reported a decrease in total oxidant status (TOS) and OSI in patients given antidepressants for 12 weeks, but an increase in TAC. In contrast, Kotan et al. ${ }^{14}$ found that treatment for 24 weeks resulted in decreased TAC. The aim of the present study was to evaluate TAA in patients with MDD and to examine the effect of antidepressant treatment on TAA. This is a follow-up of our previous study describing the novel approach of using potentiometry to measure TAA in patients with major depression.

\section{METHODS}

\section{Subjects}

Twenty-eight patients diagnosed with MDD according to the Diagnostic and Statistical Manual of Mental Disorders, fourth edition (DSM-IV), were included in this study. They had not been taking antidepressants for at least three months previously. Patients were excluded if they could not complete a self-rating scale for disturbance of cognitive function, if they had experienced an acute inflammatory state within the two weeks prior to the study, if they had an alcohol or drug dependence disorder, if they had an autoimmune disease, or if they were pregnant. The control group comprised 31 healthy individuals matched for gender, drinking and smoking status. Informed consent was obtained from all participants. The protocol for the trial was submitted to the Ethical Committee of Chuncheon Sacred Heart Hospital. The Hamilton Depression Rating Scale ${ }^{24}$ and the Beck Depression Inventory (BDI) $)^{25}$ were applied to patients at baseline and at four and eight weeks after study commencement.

\section{Antidepressant treatment}

Antidepressant drugs were chosen for each patient according to the results of psychiatric examination. The drugs were escitalopram 17.6 $\pm 4.4 \mathrm{mg} /$ day $(\mathrm{n}=17)$, duloxetine $50 \pm 17.3$ $\mathrm{mg} /$ day $(\mathrm{n}=3)$, paroxetine $14.3 \pm 4.7 \mathrm{mg} /$ day $(\mathrm{n}=7)$, and fluoxetine $20 \mathrm{mg} /$ day $(\mathrm{n}=1)$, and all were used at standard antidepressant doses.

\section{Sample preparation and analysis}

Blood samples were withdrawn from the antecubital vein between 9 am and 11 am and were processed in the laboratory immediately after collection. Serum was collected after centrifuging twice for five minutes. To calculate serum TAA, a potentiometry method was used. The standard solution (mediator system) consisted of $0.01 \mathrm{M} \mathrm{K}_{3}\left[\mathrm{Fe}\left(\mathrm{CN}_{6}\right)\right]$ and $0.1 \mathrm{mM}$ $\mathrm{K}_{4}\left[\mathrm{Fe}\left(\mathrm{CN}_{6}\right)\right]$ in a phosphate buffer solution of $\mathrm{pH}$ 7.2. A platinum electrode (active area: $0.2 \mathrm{~cm}^{2}$ ) and an $\mathrm{Ag} / \mathrm{AgCl}$ electrode were used as the working electrode and reference electrode, respectively. The value of TAA was calculated from the redox potential difference of the mediator system $\mathrm{K}_{3}\left[\mathrm{Fe}(\mathrm{CN})_{6}\right] /$ $\mathrm{K}_{4}\left[\mathrm{Fe}(\mathrm{CN})_{6}\right]$ using an antioxidant activity measuring device (MPA-1, IVA Co. Ltd., Yekaterinburg, Russia). The period of measurement was five to ten minutes. The same procedures were performed for all subsequent samples, obtained at visits in the fourth and eighth weeks.

\section{Statistical analysis}

All statistical analyses were performed with SPSS (version 17.0; SPSS, Inc., Chicago, IL, USA), with an alpha level of $<0.05$. Continuous variables were expressed as mean \pm standard deviation. Categorical variables were expressed as frequency. We utilized independent $t$-tests and analysis of variance (ANOVA) to compare means and ratios between controls and MDD patients. Correlations between variables were tested using Pearson and Spearman correlation coefficients. Discriminant analysis of the baseline TAA in the response and non-response groups was done with dBSTAT 5.0 (Chuncheon, Korea).

\section{RESULTS}

Table 1 shows that the mean BDI score was higher in the

Table 1. Demographics, mean depression scale scores and total antioxidant activity in individuals with major depressive disorder (MDD) and controls

\begin{tabular}{lcc}
\hline & Control $(\mathrm{N}=31)$ & MDD $(\mathrm{N}=28)$ \\
\hline Age (years) & $38.0 \pm 13.8$ & $35.0 \pm 14.5$ \\
Sex (female/male) & $15 / 16$ & $16 / 12$ \\
BDI (mean $\pm S D)$ & $5.8 \pm 4.6$ & $28.3 \pm 9.7^{*}$ \\
HAMD (mean $\pm S D)$ & - & $28.4 \pm 10.3$ \\
TAA (mean $\pm S D)$ & $1.35 \pm 0.38$ & $1.05 \pm 0.38^{* *}$ \\
\hline
\end{tabular}

${ }^{*} \mathrm{p}<0.001,{ }^{* *} \mathrm{p}<0.01$, independent t-test. BDI: Beck Depression Inventory (point score), HAMD: Hamilton Depression Rating Scale (point score), TAA: total antioxidant activity (mmol-eq/L) 
MDD group than in the control group (28.3 \pm 9.7 vs. $5.8 \pm 4.6$, $\mathrm{p}<0.001)$. On the other hand, the average value of TAA in the MDD group was lower $(1.05 \pm 0.38$ vs. $1.35 \pm 0.38, \mathrm{p}<0.01)$. We found that while BDI scores were negatively correlated with TAA $(r=-0.383, p<0.01)$, there was no significant correlation between HAMD scores and TAA (Figure 1).

We subdivided the MDD group according to the response to antidepressant treatment. There were 16 patients whose HAMD score decreased by over 50\% after four weeks (response group) and 12 patients whose HAMD score changed by less than $50 \%$ (non-response group). In addition, the BDI scores were not improved after eight weeks in the non-response group (Figure 2A and B). We evaluated TAA levels in the two groups after four and eight weeks of antidepressant treatment (Figure 2C). When we compared the MDD and control groups, the former showed a significantly lower TAA level at baseline. However, when we classified the MDD group according to response and then compared the three groups (control, response and non-response) using one-way ANOVA, there was no significant difference in TAA between the control

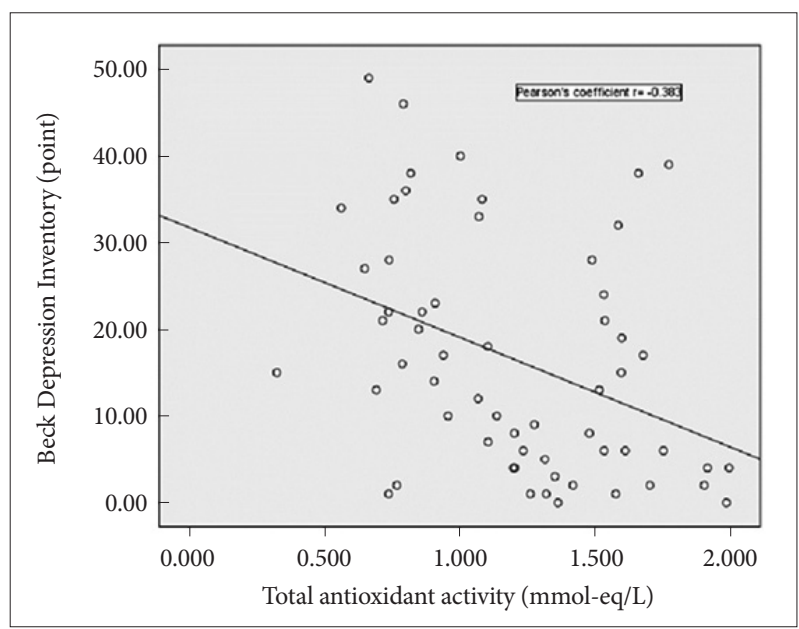

Figure 1. Plot of Beck Depression Inventory score versus total antioxidant activity. Linear associations were tested using Pearson's correlation analysis $(p<0.01)$. and response groups, while the non-response group showed significantly lower levels of TAA than the other groups ( $\mathrm{p}<$ 0.005). After four weeks, TAA in both the response and non-response groups was lower than in controls $(\mathrm{p}<0.05, \mathrm{p}<0.005$, respectively). After eight weeks, the TAA level had increased in both MDD subgroups such that there was no significant difference among the three groups.

Discriminant analysis showed that the mean pre-treatment TAA level was 1.15919 in the response group $(n=15)$ and 0.90358 in the non-response group $(\mathrm{n}=13)$. Responsivity was determined at four weeks after treatment. Wilks' lambda was 0.88735 and the $\mathrm{F}$ value was 3.30 ( $\mathrm{p}=0.0808$ ). Fisher's linear discriminant function was as follows:

$\mathrm{Z}=1.88322 \times \mathrm{B}-1.94233$

$B$ is the TAA of MDD patients before treatment. A positive $Z$ value indicates the response group; a negative value indicates the non-response group. The discriminant power of the response group was $50.0 \%$ and that of the non-response group was $75.0 \%$. The total discriminant power was $60.7 \%$.

\section{DISCUSSION}

The major findings of the present study were as follows: 1) There was a significant negative correlation between the subjective severity of depressive disorder and the TAA level; 2) TAA was significantly lower in the MDD group than in controls; 3) MDD patients who were non-responders to antidepressant treatment showed lower levels of TAA at baseline, while responders showed no significant difference to controls at baseline; and 4) After eight weeks of antidepressant treatment, TAA in both response and non-response groups was not significantly different to controls.

Bilici et al. ${ }^{17}$ have previously reported a positive correlation between the severity of depression and erythrocyte SOD and MDA levels. Similarly, Sarandol et al. ${ }^{18}$ found a positive correlation between the severity of the disease and SOD activity, suggesting that pro-oxidative challenge is higher in severe de-
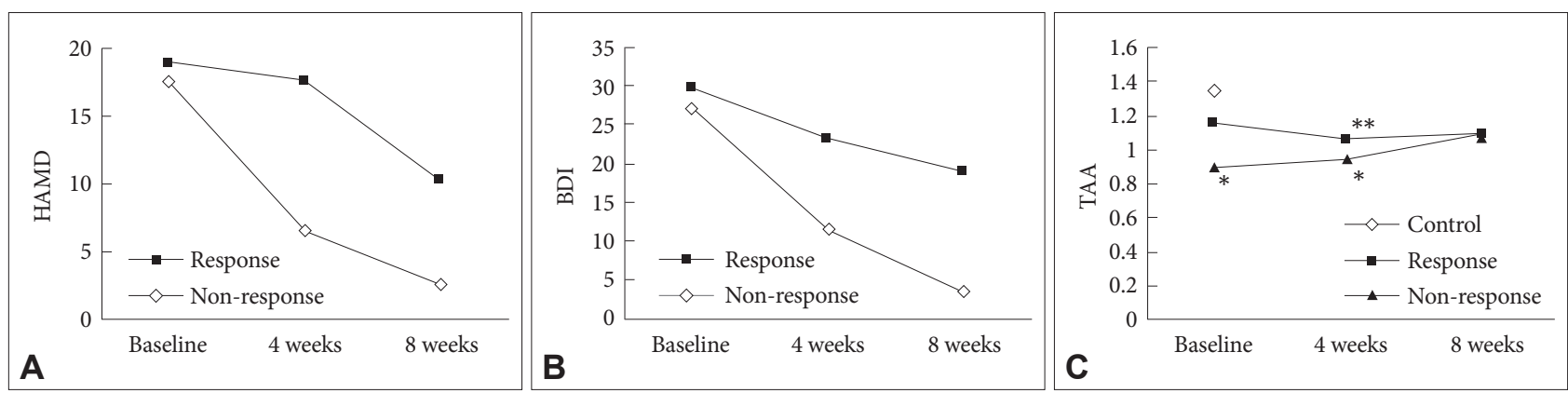

Figure 2. Hamilton Depression Rating Scale score (A), Beck Depression Inventory score (B), and total antioxidant activity levels (C) in response and non-response groups. ${ }^{*} p<0.005,{ }^{* *} p<0.05$. HAMD: Hamilton Depression Rating Scale (point score), BDI: Beck Depression Inventory (point score), TAA: total antioxidant activity (mmol-eq/L). 
pression than in the mild and moderate depression. Cumurcu et al..$^{15}$ showed a positive correlation between the severity of disease and serum TOS and OSI, while there was a negative correlation between the severity of disease and serum TAC. In the present study, we found a negative correlation between the severity of disease and TAA levels; specifically, there was a correlation of TAA with BDI scores, but not with HAMD scores. In the East Asian cultural context, MDD patients typically report somatic symptoms when they suffer from depression. ${ }^{26}$ We suggest that these physical symptoms might be more reflective of the subjective severity than the objective severity of depressive illness. TAA levels might be an objective marker of both physiological status as well as psychological (i.e., depressive) status in these individuals.

Sarandol et al. ${ }^{18}$ measured pre- and post-antidepressant treatment serum TAC for the first time, finding that pre-treatment serum TAC was lower than in the control group. However, six weeks of antidepressant treatment with either reboxetine, sertraline, or venlafaxine did not change TAC or oxidative-antioxidative system parameters such as $\mathrm{MDA}$, erythrocyte $\mathrm{SOD}$ activity, Vitamin E, C, or total carotenoid levels and GSH-Px. In line with this study, Galecki et al. ${ }^{16}$ also showed lower levels of TAC before treatment in subjects with major depressive disorder. Following 12 weeks with fluoxetine treatment, TAC values were significantly lower than in controls but there were no significant differences when measured before and after treatment. In contrast to these two studies, Cumurcu et al..$^{15}$ found increased TAC following 12 weeks of selective serotonin reuptake inhibitor treatment (i.e., escitalopram, paroxetine, or sertraline). On the other hand, Kotan et al..$^{14}$ reported no difference in TAC between control and MDD patients before various antidepressant treatments (i.e., venlafaxine, paroxetine, escitalopram, sertraline, citalopram, milnacipran, fluoxetine, tianepin, or moclobemid). In that study, after 24 weeks, the TAC level of MDD patients was significantly lower than that in the control group. In the present study, TAA was significantly lower in the non-response MDD group than in controls. Following eight weeks of antidepressant treatment, there was no significant difference between the control and non-response MDD groups.

The decreased TAA level might indicate that the mechanism of defense from oxidative stress is insufficient in patients with MDD, as suggested by Kotan et al. However, when we categorized patients with depression into two groups according to the response to antidepressant treatment, the response group didn't show any significant difference in TAA level to the control group before treatment, while the non-response group showed a significant decrease in TAA level. This finding suggests that individuals that respond clinically to antidepressant treatment retain comparable protective mechanisms against oxidative stress to the control group, compared with in- dividuals who do not respond to antidepressant treatment. Cumurcu et al suggested that oxidative and anti-oxidative activity may not change following antidepressant treatment for a relatively short period of six weeks, and that long-term treatment of at least 12 weeks is needed for oxidative and anti-oxidative system parameters to be changed. The results of the present study showing that eight weeks of antidepressant treatment changed the TAA level in the non-response MDD group suggest that at least eight weeks of treatment may be needed for any change.

If we measure TAA by potentiometry in people with MDD prior to treatment, we might be able to predict the responsiveness of patients to medication. The discriminant power of our results was insufficient to predict the responsivity to anti-depressant drugs in MDD patients; however, the discriminant power of $75.0 \%$ for the non-response group might be a clue to predicting the therapeutic effect of the drugs in clinical practice.

There are some limitations to this study. First, the sample size was relatively small. Second, in assessing the effects of antidepressant treatment, it should be noted that patients took different kinds of antidepressants. Third, there could be some confounding factors related to each patient's lifestyle, since exercise and diet may affect the level of TAA. Further studies are required to investigate the effects of antidepressants on oxidative-antioxidative systems in MDD using large samples of both response and non-response groups taken after various durations of treatment. Furthermore, there is growing emphasis on the importance of lifestyle modifications such as changes to exercise or diet in addition to antidepressant treatment and/or psychotherapy in MDD patients. Future studies should also investigate the association between supplementation with antioxidant foods or exercise and antidepressant treatment response and TAA in MDD patients.

\section{Acknowledgments}

This work was supported by the Korea Atomic Energy Research Institute Project and the Hallym University Research Fund (HRF-G-2015-4).

\section{REFERENCES}

1. Kessler RC. The effects of stressful life events on depression. Annu Rev Psychol 1997;48:191-214.

2. Post RM. Transduction of psychosocial stress into the neurobiology of recurrent affective disorder. Am J Psychiatry 1992;149:999-1010.

3. Scapagnini G, Davinelli S, Drago F, De Lorenzo A, Oriani G. Antioxidants as antidepressants: fact or fiction? CNS Drugs 2012;26:477-490.

4. Belmaker RH, Agam G. Major depressive disorder. N Engl J Med 2008; 358:55-68.

5. Maes M, Galecki P, Chang YS, Berk M. A review on the oxidative and nitrosative stress (O\&NS) pathways in major depression and their possible contribution to the (neuro) degenerative processes in that illness. Prog Neuropsychopharmacol Biol Psychiatry 2011;35:676-692.

6. Gawryluk JW, Wang JF, Andreazza AC, Shao L, Young LT. Decreased levels of glutathione, the major brain antioxidant, in post-mortem prefrontal cortex from patients with psychiatric disorders. Int J Neuropsy- 
chopharmacol 2011;14:123-130.

7. Raha S, Robinson BH. Mitochondria, oxygen free radicals, and apoptosis. Am J Med Genet 2001;106:62-70.

8. Aksenova MV, Aksenov MY, Mactutus CF, Booze RM. Cell culture models of oxidative stress and injury in the central nervous system. Curr Neurovasc Res 2005;2:73-89.

9. Berk M, Ng F, Dean O, Dodd S, Bush AI. Glutathione: a novel treatment target in psychiatry. Trends Pharmacol Sci 2008;29:346-351.

10. Filomeni G, Ciriolo MR. Redox control of apoptosis: an update. Antioxid Redox Signal 2006;8:2187-2192.

11. Halliwell B. Phagocyte-derived reactive species: salvation or suicide? Trends Biochem Sci 2006;31:509-515.

12. Halliwell B. Reactive species and antioxidants. Redox biology is a fundamental theme of aerobic life. Plant Physiol 2006;141:312-322.

13. Lee SY, Lee SJ, Han C, Patkar AA, Masand PS, Pae CU. Oxidative/nitrosative stress and antidepressants: targets for novel antidepressants. Prog Neuropsychopharmacol Biol Psychiatry 2013;46:224-235.

14. Kotan VO, Sarandol E, Kirhan E, Ozkaya G, Kirli S. Effects of longterm antidepressant treatment on oxidative status in major depressive disorder: a 24-week follow-up study. Prog Neuropsychopharmacol Biol Psychiatry 2011;35:1284-1290.

15. Cumurcu BE, Ozyurt H, Etikan I, Demir S, Karlidag R. Total antioxidant capacity and total oxidant status in patients with major depression: impact of antidepressant treatment. Psychiatry Clin Neurosci 2009;63:639-645.

16. Galecki P, Szemraj J, Bienkiewicz M, Florkowski A, Galecka E. Lipid peroxidation and antioxidant protection in patients during acute depressive episodes and in remission after fluoxetine treatment. Pharmacol Rep 2009;61:436-447.

17. Bilici M, Efe H, Koroglu MA, Uydu HA, Bekaroglu M, Deger O. Anti- oxidative enzyme activities and lipid peroxidation in major depression: alterations by antidepressant treatments. J Affect Disord 2001;64:43-51.

18. Sarandol A, Sarandol E, Eker SS, Erdinc S, Vatansever E, Kirli S. Major depressive disorder is accompanied with oxidative stress: short-term antidepressant treatment does not alter oxidative-antioxidative systems. Hum Psychopharmacol 2007;22:67-73.

19. Tarpey MM, Wink DA, Grisham MB. Methods for detection of reactive metabolites of oxygen and nitrogen: in vitro and in vivo considerations. Am J Physiol Regul Integr Comp Physiol 2004;286:R431-R444.

20. Sofic E, Rustembegovic A, Kroyer G, Cao G. Serum antioxidant capacity in neurological, psychiatric, renal diseases and cardiomyopathy. J Neural Transm 2002;109:711-719.

21. Erel O. A novel automated direct measurement method for total antioxidant capacity using a new generation, more stable ABTS radical cation. Clin Biochem 2004;37:277-285.

22. Brainina Kh Z, Ivanova AV, Sharafutdinova EN, Lozovskaya EL, Shkarina EI. Potentiometry as a method of antioxidant activity investigation. Talanta 2007;71:13-18.

23. Lee GJ, Lee SK, Kim JM, Rhee CK, Lee YK, Brainina KZ, et al. Application feasibility of antioxidant activity evaluation using potentiometry in major depressive disorder. Electrochemistry 2014;82:264-266.

24. Hamilton M. Development of a rating scale for primary depressive illness. Br J Soc Clin Psychol 1967;6:278-296.

25. Beck AT, Ward CH, Mendelson M, Mock J, Erbaugh J. An inventory for measuring depression. Arch Gen Psychiatry 1961;4:561-571.

26. Zhou X, Min S, Sun J, Kim SJ, Ahn JS, Peng Y, et al. Extending a structural model of somatization to South Koreans: cultural values, somatization tendency, and the presentation of depressive symptoms. J Affect Disord 2015;176:151-154. 